Revue scientifique sur la conception et l'aménagement de l'espace

\title{
L'architecte paysagiste à Alger pendant la période coloniale (1830-1962) : une figure émergente
}

The Landscape Architect in Algiers During the Colonial Period (1830-1962) : An

Emerging Figure

\section{Fares Trodi, Nadia Djelal et Philippe Potié}

\section{(2) OpenEdition}

Journals

Édition électronique

URL : http://journals.openedition.org/paysage/10368

DOI : $10.4000 /$ paysage. 10368

ISSN : 1969-6124

Éditeur :

École nationale supérieure du paysage de Versailles-Marseille, Institut national des sciences appliquées Centre Val de Loire - École de la nature et du paysage, École nationale supérieure d'architecture et de paysage de Bordeaux, École nationale supérieure d'architecture et de paysage de Lille, Agrocampus Angers

\section{Référence électronique}

Fares Trodi, Nadia Djelal et Philippe Potié, «L'architecte paysagiste à Alger pendant la période coloniale (1830-1962) : une figure émergente », Projets de paysage [En ligne], 13 | 2015, mis en ligne le 31 décembre 2015, consulté le 04 septembre 2020. URL : http://journals.openedition.org/paysage/ 10368 ; DOI : https://doi.org/10.4000/paysage.10368

Ce document a été généré automatiquement le 4 septembre 2020

Projets de paysage 


\section{L'architecte paysagiste à Alger pendant la période coloniale (1830-1962) : une figure émergente}

The Landscape Architect in Algiers During the Colonial Period (1830-1962) : An Emerging Figure

Fares Trodi, Nadia Djelal et Philippe Potié

1 Quels rôles ont joué les concepteurs paysagistes dans les différentes politiques urbaines de l'Algérie au cours de la période coloniale ? Ces professionnels, qui commençaient à émerger dans les villes françaises à la même époque, ont-ils existé en Algérie?

2 Nous essayerons dans cet article de rappeler d'abord les moments marquants de l'histoire générale des architectes paysagistes depuis le $\mathrm{XIX}^{\mathrm{e}}$ siècle (en termes de formation et d'évolution du métier de paysagiste), puis des pratiques urbaines impliquant des figures professionnelles militaires ou civiles (architectes, urbanistes, ingénieurs) qui ont joué le rôle de concepteur paysagiste dans l'Algérie coloniale. Nous défendrons enfin l'idée que l'espace urbain algérois a hérité des espaces paysagers produits pendant cette période par le biais des multiples politiques publiques d'aménagement, notamment d'embellissement réglementaire. La profession de paysagiste concepteur n'y a pas été organisée, mais les compétences requises dans ce domaine ont pu émerger pendant la période coloniale comme d'ailleurs en métropole à la même époque.

\section{Une petite histoire des paysagistes}

3 L'analyse du rôle des concepteurs paysagistes dans l'aménagement urbain des villes algériennes, notamment à Alger, nécessite de rappeler d'abord l'évolution du métier des paysagistes français en métropole et leur apport dans l'aménagement urbain en Algérie coloniale de 1830 à 1962. Il s'agira ainsi de comprendre l'héritage (formations, compétences, etc.) de la profession de paysagiste dans l'Algérie postcoloniale. Nous 
nous appuierons principalement sur les publications de l'historien des jardins JeanPierre Le Dantec (2002) et de Zohra Hakimi (2011).

\section{Paysagiste : un essai de définition}

En 1828, l'ouvrage de Gilbert Laing Meason (1769-1832), On the landscape architecture of the great painters of Italy, désigne les landscape designers et les landscape architects, comme les figures praticiennes, héritières des peintres de paysage depuis la Renaissance, et chargées de concevoir la composition dite paysagère des jardins privés et publics. Le terme d'architecte paysagiste apparaît en France à la même époque (Donadieu, 2009).

5 Se référant souvent au maître jardinier André Le Nôtre (1613-1700), les concepteurs français de jardins étaient souvent des dessinateurs et des réalisateurs de jardins privés et publics dont le savoir-faire comprenait, outre la connaissance horticole des végétaux, les techniques et les principes de leur ordonnancement dans l'espace (Chaux, 1989). Après la Seconde Guerre mondiale, les paysagistes ${ }^{1}$ furent d'abord et surtout des planteurs de végétaux et des réalisateurs d'espaces verts, de parcs et de jardins avant qu'ils ne se transforment après 1960 en professionnels organisés avec parfois une double formation de paysagiste et d'urbaniste (Dubost, 1983).

6 Aujourd'hui, les paysagistes concepteurs sont requis par les maîtres d'ouvrage publics et privés comme maîtres d'œuvre de projets et d'études d'aménagement, de l'échelle du territoire à celle du jardin (Fédération française du paysage, 2009). Ils développent une profession qui cherche à valoriser les lieux « à des fins fonctionnelles, esthétiques et soutenables; mais aussi de façon appropriée aux divers besoins écologiques et humains des générations actuelles et futures » (définition donnée par l'International Federation of Landscape Architects - IFLA - en 2007).

7 Ces compétences de concepteurs et d'ingénieurs dépendent fondamentalement des écoles de formation qui délivrent des diplômes de paysagistes (sept en France en 2015²).

\section{La formation des paysagistes en France}

8 Apparue au xIX siècle, la distinction entre les architectes paysagistes (landscape architects) - des concepteurs maîtres d'œuvre - et les jardiniers (gardeners ${ }^{3}$ ) - des techniciens du jardinage - a différencié les écoles de paysagistes au cours du $\mathrm{xx}^{\mathrm{e}}$ siècle. D'un côté les sciences et les techniques de l'horticulture et de l'agronomie (comme à l'École d'horticulture de Versailles depuis 1873) et de l'autre les arts de l'architecture de paysage et du design paysagiste (comme au département d'architecture de l'université d'Harvard depuis 1899) (Donadieu, 2009).

9 En France, les paysagistes ont été, jusqu'à la dernière guerre, à la fois des dessinateurs de jardins, des ingénieurs, des entrepreneurs et des pépiniéristes formés en général à l'École d'horticulture de Versailles. Ils ont conquis ensuite, comme dans les métiers de l'architecture, un statut de professionnels ${ }^{4}$ organisés (Cicé et Dubost, 1986).

10 À Versailles, jusqu'en 1945, ces praticiens concepteurs, autodidactes ou ingénieurs en horticulture, ont d'abord été appelés maître-jardinier, jardiniste, architecte de jardin et architecte paysagiste. Puis à partir de 1946, leur a été délivré le certificat de paysagiste, ensuite le titre de paysagiste (après l'accomplissement d'un stage de deux ans) diplômé par le ministère de l'Agriculture ${ }^{5}$ après formation dans la section du paysage et de l'art 
des jardins de l'École nationale d'horticulture de Versailles. La création de l'Ordre des architectes en 1940 a abouti à l'interdiction, jusqu'à aujourd'hui, aux paysagistes concepteurs le port du titre d'architecte paysagiste (Blanchon, 2007). À partir de 1962, leur diplôme a été celui de paysagiste diplômé par le gouvernement (DPLG) ou d'ingénieur paysagiste selon leur formation respectivement dans les Écoles nationales supérieures d'horticulture de Versailles ou d'Angers. La section du paysage et de l'art des jardins de l'École nationale supérieure d'horticulture est devenue en 1976 l'actuelle École nationale supérieure de paysage qui délivrera à partir de 2018 le diplôme d'État de paysagiste (abandon du diplôme de paysagiste DPLG) ${ }^{6}$.

11 Le diplôme français de paysagiste DPLG est identique au titre professionnel d'architecte paysagiste qui est donné, en général, dans une quarantaine de pays, par les organisations professionnelles d'architectes paysagistes reconnues par la Fédération internationale des architectes paysagistes (Donadieu, 2014).

Figure 1. Chronologies de l'évolution des diplômes à Versailles

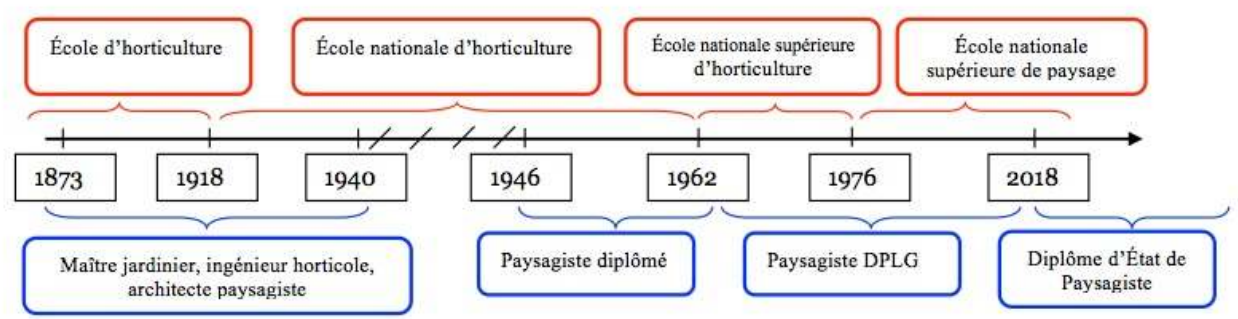

\section{L'évolution de la figure de paysagiste en France}

12 Comme le précise l'historien des jardins Michel Racine (2002), jusqu'au XIX siècle, la commande quasi unique était celle des jardins et des parcs appartenant aux familles royales et aristocratiques. Elle concernait des architectes, autant que des jardiniers et des peintres et, le plus souvent, des autodidactes. Dès la fin du XIX siècle, le paysagiste a contribué à l'organisation des agglomérations, à l'embellissement de l'espace public et à la création de villes assainies et «verdoyantes». Il a créé les parcs et les plantations d'arbres dans les villes comme à Paris avec l'ingénieur Adolph Alphand (1817-1891) et aux États-Unis (Central Park, New York) avec le paysagiste Frederic Law Olmsted (1822-1903). Au Maroc la planification des villes européennes du protectorat français a été confiée à l'architecte paysagiste Jean Claude Nicolas Forestier (1861-1930) et à l'urbaniste Henri Prost (1874-1959), notamment à Rabat entre 1912 et 1930 (Bennani, 2006).

13 À la fin de la Première Guerre mondiale, le développement des villes et leur embellissement grâce à la loi Cornudet ${ }^{7}$ de 1919 diversifient le métier de paysagiste avec l'aménagement des parcs et des jardins publics. À partir de 1945, il commence à se transformer en profession organisée de plus en plus orientée vers l'urbanisme. Toutefois, pendant cette période de la reconstruction d'après-guerre (1950-1980) «on peut dire que la mission des paysagistes s'est surtout bornée à "mettre du vert" dans les espaces interstitiels des grands ensembles urbains»(Dubost, 1983), en dépit de réalisations remarquables aujourd'hui redécouvertes (Blanchon, 2007). Le jardin urbain peu fonctionnel, trop cher et sophistiqué, a dû céder la place au simple verdissement, 
privilégiant les conceptions des ingénieurs sur celles des «hommes de l'art» (Le Dantec, 2002).

Dans les années 1970, apparait une nouvelle notion liée à l'évolution rapide des paysages ruraux : «ce qu'ils appellent le "grand paysage", ce n'est plus seulement le végétal comme spécificité d'un matériau, c'est le sol, c'est l'air, la lumière, tout ce qui est vivant»(Dubost, 1983). La rupture relative avec les doctrines du Mouvement moderne (CIAM) va se traduire à la fin des années 1970 par la création d'une mission du paysage au sein du jeune ministère de l'Environnement (1972). Jusqu'en 1997, elle agira de façon interministérielle pour permettre de renouer des relations entre habitants, sites et paysages. Grâce à l'esquisse d'un mouvement de planification écologique et environnementale dans les années 1970, est ainsi amorcée une pratique de projet à l'échelle des territoires administrés. Elle ne prendra réellement forme qu'avec la loi dite Paysage de janvier 1993, et la réalisation des atlas de paysage dans toutes les régions françaises, des plans de paysage à l'échelle des collectivités et des chartes de paysage, notamment dans les Parcs naturels régionaux.

15 À la fin des années 1970, et avec le développement dans les formations comme dans les actions publiques de la notion de projet de paysage, les professionnels du paysage vont développer le métier de concepteur de projet, à travers la recherche des formes pour répondre à des fonctions prévues par un programme. Cette pensée s'est élargie "d'espaces limités comme les "espaces d'accompagnement" (des voies publiques, des parkings, des places, des terrains des jeux et de sport, etc.), les jardins publics et les parcs, à l'espace tout entier quand il s'agit d'études d'impact ou d'études de site dans un grand projet d'aménagement régional » (Dubost, 1983).

C'est dans le cadre du début de cette évolution historique de la figure professionnelle du paysagiste en France que la dimension paysagère de l'urbanisme en Algérie coloniale va émerger. La ville d'Alger, en tant que première ville conquise par les Français, a vu d'importantes actions de mise et de remise en œuvre de son espace urbain public que nous analyserons précisément dans le prochain chapitre.

\section{Paysagistes et urbanistes à Alger (1830-1930)}

17 De 1830 à 1930, la politique coloniale va profondément marquer les villes algériennes, d'abord de manière autoritaire, puis réglementaire.

\section{Le paysagisme militaire à Alger}

18 La colonisation de l'Algérie par les Français en 1830 fut marquée par des destructions du patrimoine architectural et urbain de l'ancienne ville d'Alger, notamment dans le quartier de la Casbah (Béguin, 1983). Des aménagements urbains se traduisirent par la construction de différents jardins publics dont celui du Hamma, décidé en 1832 par le général Avisard, et le jardin Marengo en 1833 restauré par le colonel Marengo qui lui a donné son nom (Chabbi-Chemrouk, 2003). Il remplaça le jardin du Dey d'Alger.

19 En 1837, comme le précise Zohra Hakimi (2011), l'ingénieur des Ponts et Chaussées Victor Poirel (1804-1881) sera le premier à imaginer un plan pour l'embellissement de l'ancienne ville grâce à l'aménagement des voies de Bab-Azoun et Bab el-Oued bordant la mer, ainsi que des places du Gouvernement et de la République. Cependant c'est en 
1846 qu'Alger connaîtra ses premières promenades après l'approbation du plan d'alignement de la ville élaboré par l'architecte des bâtiments civils et de la voirie Pierre Auguste Guiauchain (1806-1875). Celui-ci transforma les anciennes fortifications turques en un boulevard de 40 à 60 mètres de largeur aménagé en jardins, rampes et escaliers pour donner l'actuel boulevard Laferrière.

20 L'espace urbain public devenait ainsi plus fonctionnel et agréable pour les colons, grâce à des percées souvent accompagnées de plantations qui maintenaient l'ombre et la fraîcheur. Cette pratique va devenir une des constantes de l'urbanisme colonial (Béguin, 1983). Elle fut mise en œuvre par un service spécial des plantations créé en 1842 par le ministère de la Guerre afin de développer les plantations publiques dans toutes les régions occupées (L'Afrique du nord illustrée, 1914).

21 Il a fallu attendre le début $\mathrm{du} \mathrm{xx}^{\mathrm{e}}$ siècle pour que les pratiques radicales des tracés régulateurs que l'on pourrait qualifier de paysagisme militaire prennent une tournure différente. « Il faut, pour leur plaire [aux citadins], créer des quartiers bien aérés, ornés de tous les attraits que la nature ou l'art mettent à la disposition des architectes et des administrateurs : jardins publics et privés, ombrage dans les avenues, portiques dans les rues, etc. » (Royer et al., 1935).

\section{Des villes nouvelles européennes à Alger}

22 Au début de la colonisation, le jardin du Hamma était dirigé par le lieutenant de vaisseau Barnier qui avait succédé au commandant Bérard, auteur des premiers tracés du jardin. Ce vaste parc devient au milieu du XIX ${ }^{e}$ siècle un élément structurant de la composition de l'espace urbain algérois. Entre 1855 et 1859 plusieurs projets sont proposés pour une ville nouvelle située entre le jardin et l'ancienne ville, comme ceux du géomètre Vigouroux et du conducteur des Ponts et Chaussées Caillat en 1858, du géographe Mac Carthy et de Genevay entre 1856 et 1858 . S'y ajoute celui de l'architecte de la commune d'Alger Charles Frédéric Henri Chassériau (1802-1896) en 1858 pour Napoléon-Ville (60 000 hab.) (Béguin, 1983). Ce dernier projet comprenait douze places, huit boulevards plantés d'arbres dont un central de 45 mètres et trente rues de 19 à 14 mètres (Oulebsir, 2004).

L'ensemble de ces projets créés depuis 1830 par des architectes et des ingénieurs, pour la plupart militaires puis civils (Cohen, 2010), n'aboutira pas, malgré des attentions de composition paysagère exprimées chez les ingénieurs militaires ${ }^{8}$ (Malverti et Picard, 1989). Néanmoins ces projets ont enrichi les conceptions futures par des idées telles que les projets successifs $(1870,1907$ et 1911) des ingénieurs Jean de Redon et de son fils Eugène pour la création d'un front décoratif à Bab el-Oued, le prolongement du boulevard de front de mer du côté de Bab-Azoun, et les différents plans d'aménagement de la ville analysés plus loin.

\section{Les prémices d'une politique publique d'embellissement à Alger}

24 La ville d'Alger s'appuie sur une politique publique d'embellissement au début du $\mathrm{xx}^{\mathrm{e}}$ siècle et jusqu'en 1930 (année de la célébration du centenaire de l'occupation française), d'abord par la création le 18 mars 1904 du comité de conservation des 
édifices mauresques de la ville ancienne (Hakimi, 2011) présidé par le lieutenantcolonel en retraite E.-D. de Grammont. Favorable à cette politique, le gouverneur général Charles Célestin Auguste Jonnart (1857-1927) transmet ses directives aux architectes chargés de la construction d'édifices publics en affirmant ainsi le style néomauresque pour Alger et l'Algérie. In fine c'est la loi Cornudet de 1919 qui instaure les plans d'aménagement, d'embellissement et d'extension.

Un peu plus tôt, en 1914, ont été lancés des travaux de restauration et d'embellissement du jardin d'Essai suite à un concours entre architectes remporté par Paul Régnier et Paul Guillon. On leur doit la perspective de 500 mètres de long depuis le musée des Beaux-Arts jusqu'à la rue Sadi-Carnot, et l'ouverture en 1918 de l'École d'horticulture que le maréchal Soult réclamait depuis 1842 (Carra et Gueit, 1952), bien avant l'École d'horticulture de Versailles créée en 1873, ceci afin de développer, notamment, les espaces verts publics et privés dans les villes d'Algérie.

Figure 2. Parc de Galland (actuel parc de la Liberté), 1915

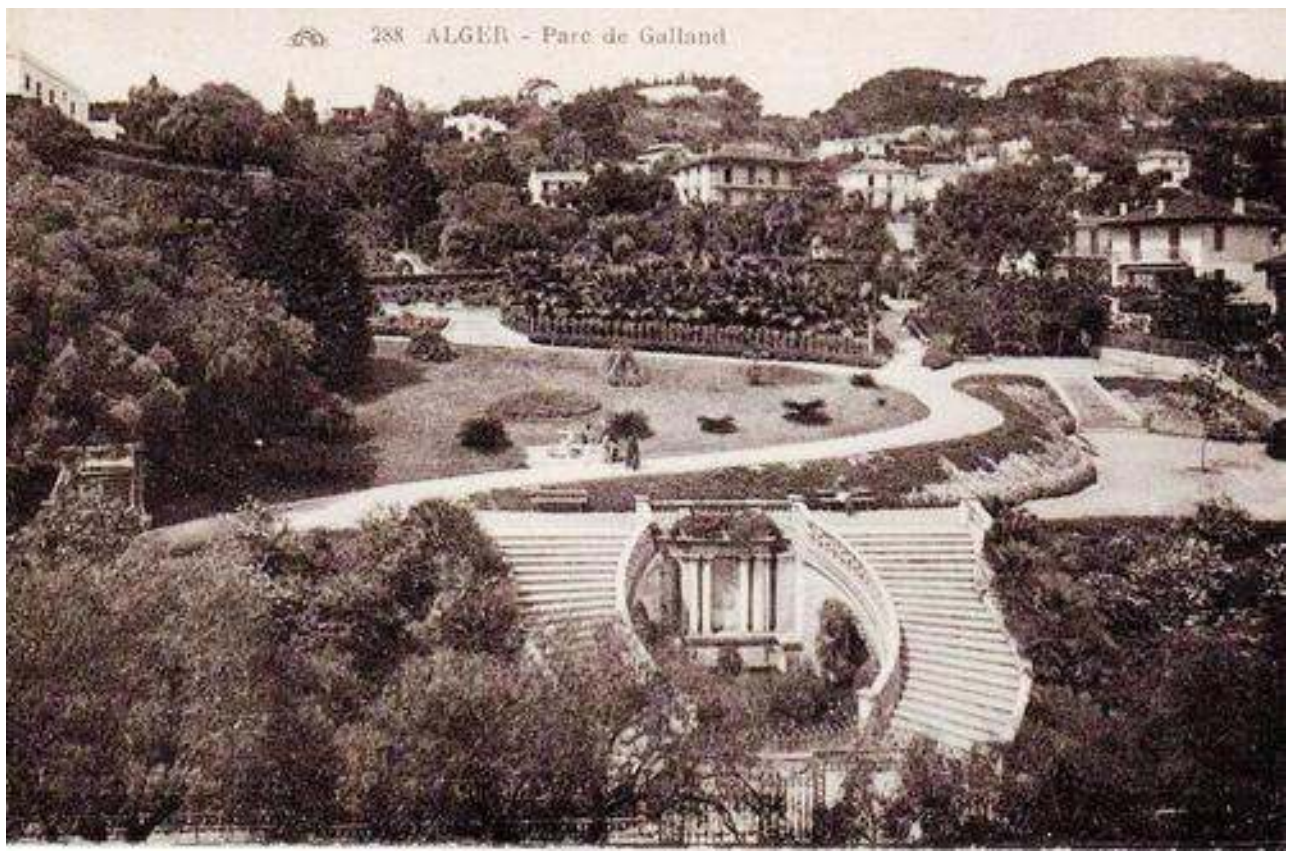

\section{Une pensée paysagiste en gestation (1930-1937)}

Entre 1925 et 1929, la ville d'Alger se dota d'un plan d'amélioration des alignements et de nivellement dit: "plan Poulain ». Il fut élaboré par César Poulain, technicien communal et chef de service de la voirie d'Alger. Mais le 18 octobre 1929 on confia l'élaboration du plan d'aménagement, d'embellissement et d'extension (PAEE) d'Alger à la Société des plans régulateurs des villes du géomètre et urbaniste René Danger (1872-1954) (Hakimi, 2011).

Cette société, comme l'indique Zohra Hakimi (2011) était composée d'architectes, d'ingénieurs, de géomètres et d'urbanistes et avait comme doctrine: assainir, ordonner, embellir, idées inspirées des cours d'urbanisme de l'architecte paysagiste français Jacques Gréber (1882-1962). Le rapport de Danger supprime l'élaboration du plan par un architecte, terme qui est remplacé par celui d'un homme de l'art tel que 
précisé dans la loi Cornudet. Il propose, entre autres suggestions, des réserves d'espaces libres et d'espaces publics, des points de vue, des belvédères et la création d'un boulevard de promenade de 60 mètres de largeur et de 860 mètres de longueur sur les fortifications de 1840, faisant la jonction des boulevards Laferrière et Guillemin. Avenues, promenades et places s'inscrivent en prolongement du système urbain, en prévoyant une large proportion de jardins et d'arbres. Les architectes tirent parti de l'idée nouvelle de cité-jardin avec des jardins publics voisins, des équipements collectifs et des places aux formes arrondies aux intersections des axes et des squares aménagés. Il est également proposé de préserver les "beautés naturelles" des coteaux de Mustapha, en intégrant dans le domaine public les réserves boisées et les belvédères, tout en limitant les hauteurs des constructions à 11,50 mètres en vue d'inciter les propriétaires à entourer leurs villas de jardins.

Des rectificatifs ont été portés sur le plan afin de supprimer certaines réserves de terrains affectées à des jardins publics. Ainsi, le plan est approuvé en 1931 et révisé en 1934 pour fusionner avec le projet régional d'urbanisme d'Alger', élaboré de 1935 à 1937 par l'urbaniste Henri Prost (qui a travaillé au Maroc avec le général Lyautey) en compagnie de l'ingénieur urbaniste Maurice Rotival (1897-1980). Prost s'intéresse particulièrement à la préservation des sites et des paysages grâce à des réserves forestières, et s'attache à ménager des vues panoramiques vers la baie d'Alger, en développant les principes formulés pour la côte varoise (Cohen, 2010).

\section{Les architectes « paysagent » Alger}

Entre 1931 et 1937 dans le cadre du PAEE et ensuite du projet régional d'urbanisme, différents projets sont animés par des ingénieurs urbanistes : Rotival et Croci, ou des architectes de l'École des beaux-arts tels que Léon Claro (1899-1991) pour son projet de reconstruction $\mathrm{du}$ quartier de l'ancienne préfecture ${ }^{10}$ adopté par une délégation composée de nombreuses professions. Pour la première fois, d'autres disciplines ${ }^{11}$ que l'architecture sont prises en considération par l'urbanisme qui devient de plus en plus l'affaire de compétences élargies (Hakimi, 2011).

Des projets comme le Foyer civique (1927-1935) sont traités en jardin : Léon Claro et l'architecte Albert Cès composent avec l'air et le soleil les abords du Foyer (Oulebsir, 2004). Mais c'est le plan de l'urbaniste Tony Socard (1901-1996) en 1933, traitant la ville comme un système de parcs et de jardins avec un boulevard promenade, ainsi que le concours d'embellissement des jardins d'Alger en 1934 avec l'architecte Raymond Taphoureau qui définissent le projet paysager pour Alger dans cette période. 
Figure 3. Tony Socard, plan d'aménagement de la ville d'Alger, 1933

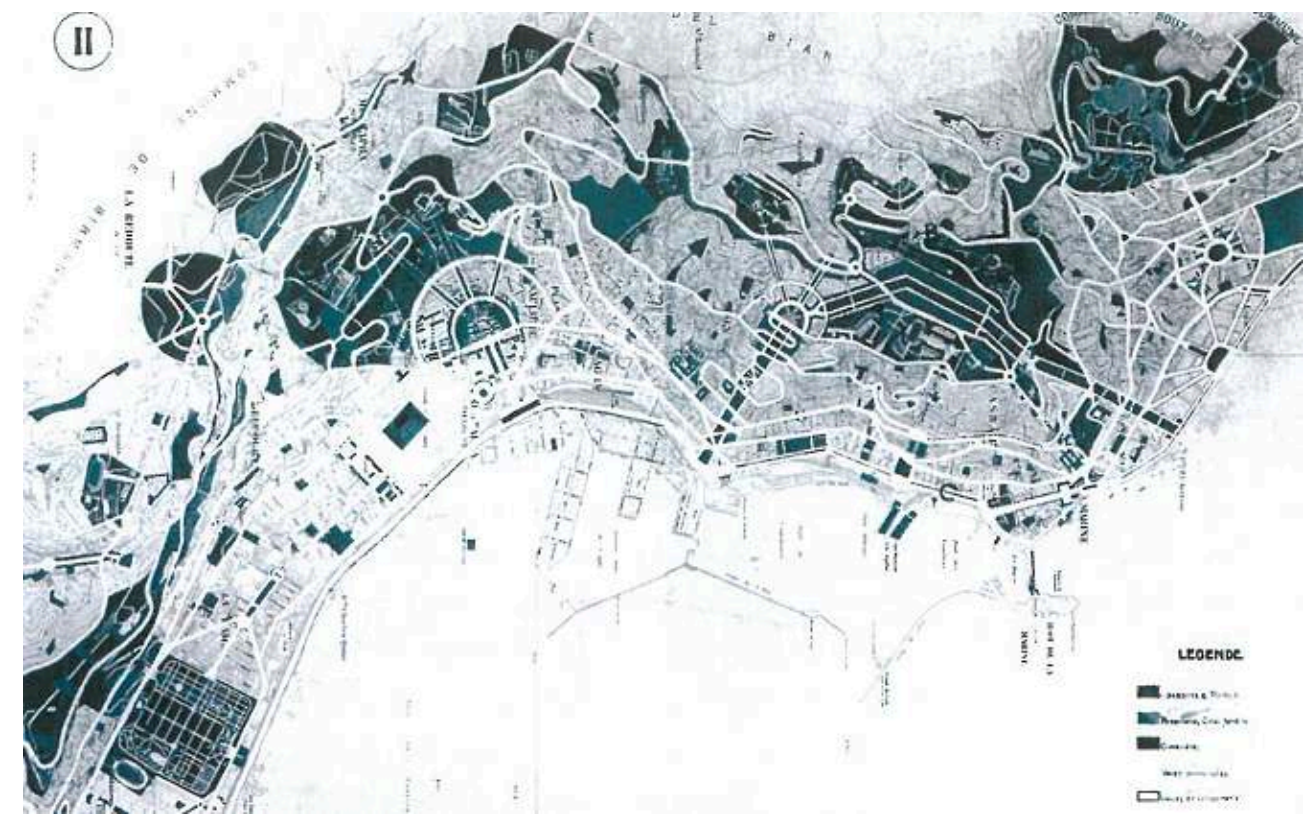

Source : Zohra Hakimi, 2011.

Figure 4. Tony Socard, Raymond Taphoureau, jardin méditerranéen, parc Mont-Riant/Saint-Saëns (actuel parc de Beyrouth)

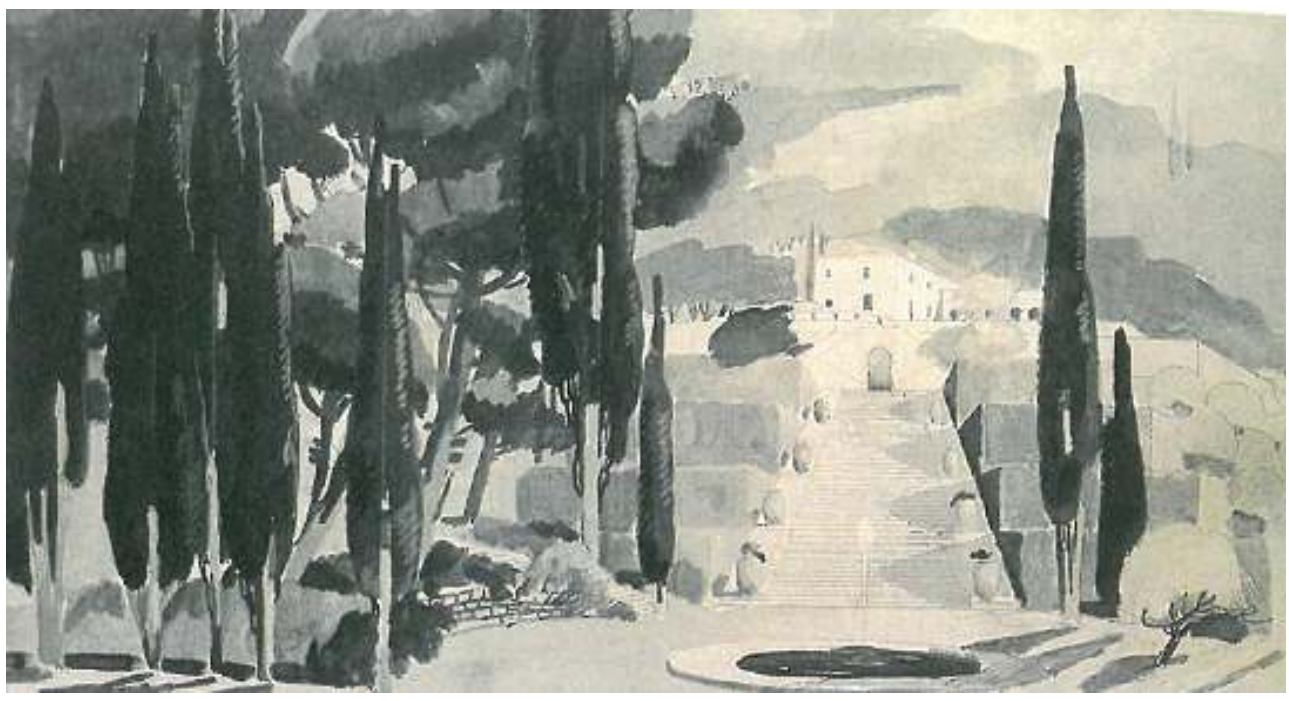

Projet primé au concours des jardins d'Alger, 1934.

Source : Naima Chabbi-Chemrouk, 2003.

31 Ces projets furent conçus par des architectes formés à l'École des beaux-arts. Ils pouvaient dessiner des parcs et des jardins publics et privés selon les modèles en vogue depuis le XIX ${ }^{e}$ siècle en France et en Europe. Ceux-ci relevaient soit du style paysager (irrégulier ou pittoresque) soit régulier, soit des deux à la fois (style mixte) ${ }^{12}$. Car, à cette période, les projets de l'École des beaux-arts imposaient, le plus souvent, de dessiner les jardins "accompagnant" le bâtiment projeté (Le Dantec, 2002). L'architecte devenait alors également un architecte de jardins. Mais ces concepteurs ne 
savaient pas s'occuper de leur gestion technique confiée à des ingénieurs en horticulture et à des maîtres et ouvriers jardiniers.

La municipalité de Charles Brunel (1929-1936) lance en 1934 un concours ouvert aux architectes et artistes paysagistes français pour la création et la transformation de jardins publics ${ }^{13}$, dont l'aménagement de l'esplanade Foch et le jardin d'enfants (1935-1941) d'après « le projet artistique » de l'architecte ingénieur Maurice Rotival ${ }^{14}$ avec le concours de l'architecte Jacques Guiauchain (1884-1960). Celui-ci renforcera le boulevard Laferrière (jardin Laferrière). Tout est pensé par l'architecte affirment les deux numéros du journal des travaux publics ${ }^{15}$ : revêtement, rampes, trottoirs, escalier, jardin, arbres, monument, place, square, perspectives et points de vue sur la baie d'Alger.

Figure 5. La perspective du projet d'ensemble de l'aménagement des boulevards Laferrière et Maréchal-Foch

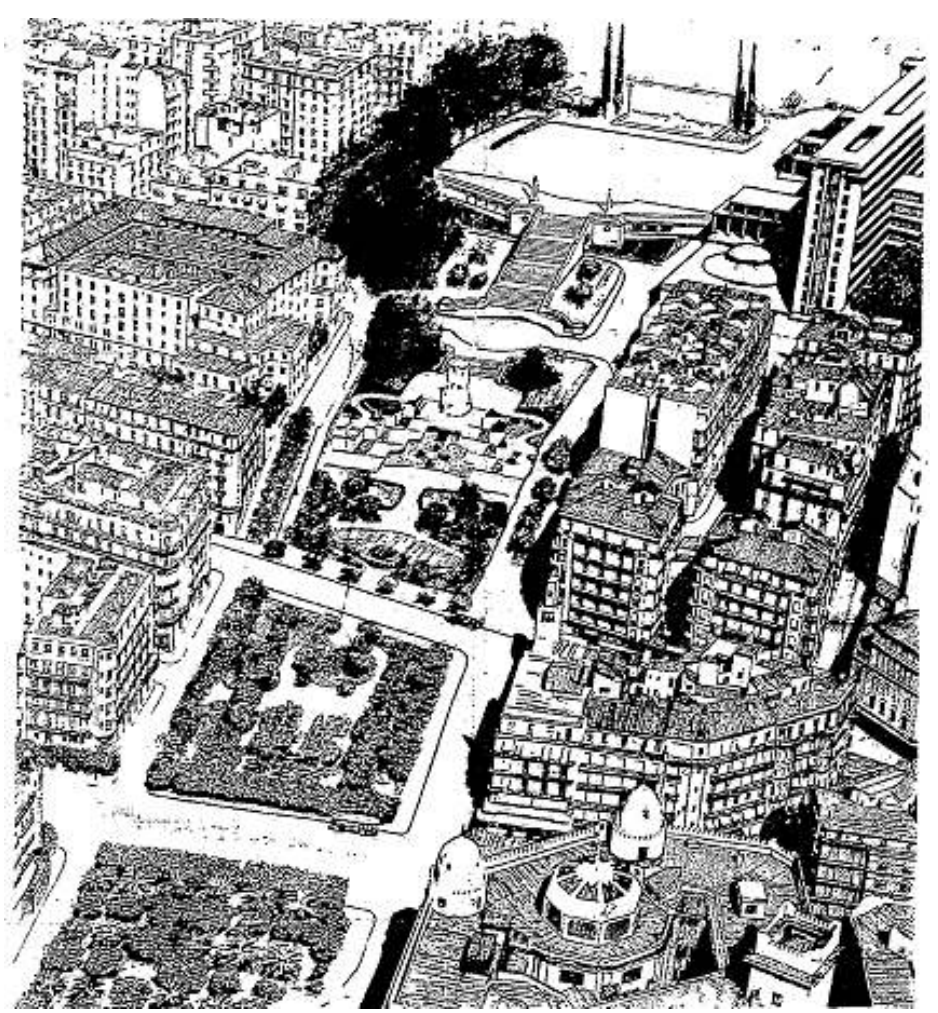

Dessin de Maurice Rotival, 1935.

Source : gallica.bnf.fr/Bibliothèque nationale de France. 


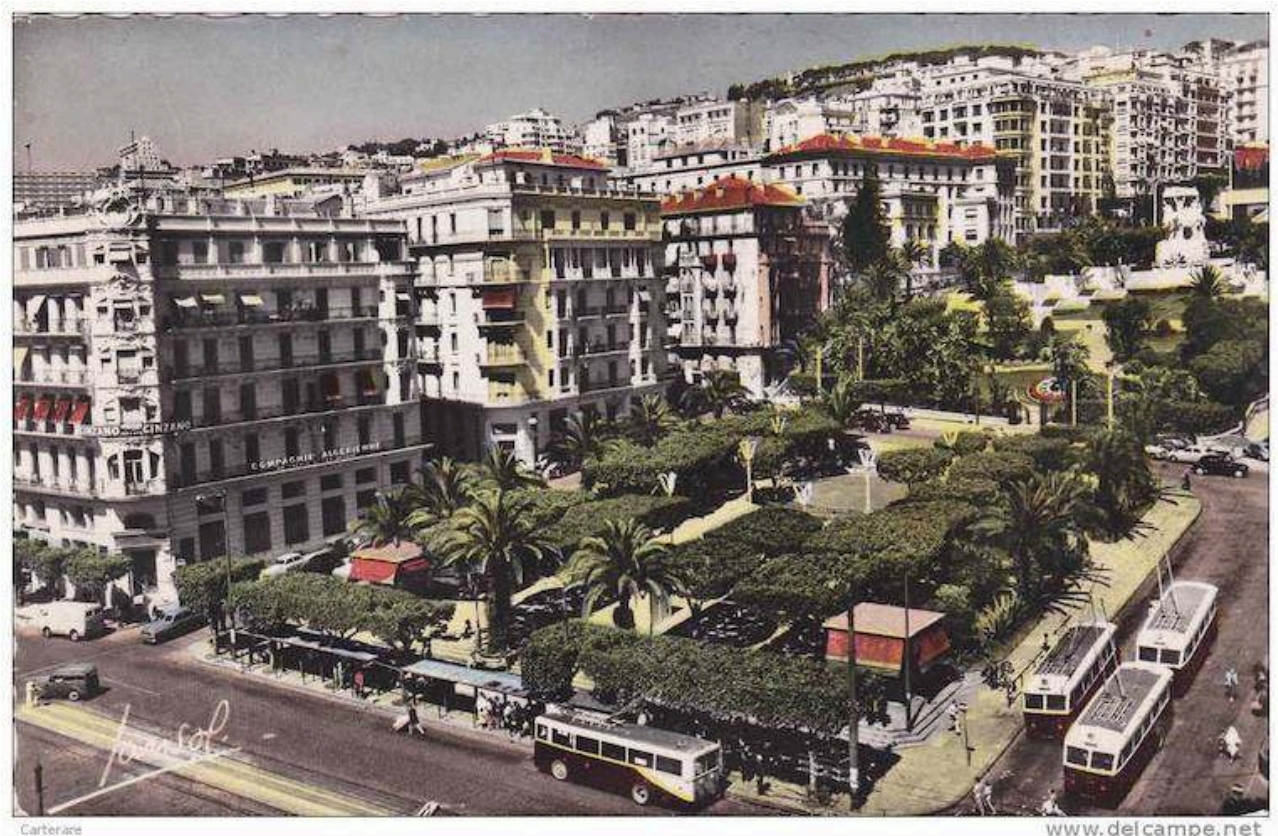

Photo anonyme, prise vers 1956

La conception du jardin prend en considération le monument aux morts conçu par Paul Landowski (1875-1961), retravaillé à l'Indépendance par l'artiste Issiakhem M'hamed (1928-1985) (Chabbi-Chemrouk, 2003). Dans le jardin, sera créée l'Horloge florale (1955) confiant son embellissement, comme tout espace urbain en Algérie, à des ouvriers jardiniers autodidactes ou formés dans les sept écoles d'agriculture ${ }^{16}$ existant à cette époque en Algérie. 
Figure 7. Des jardiniers de la ville mettent en place la décoration de l'Horloge florale du jardin Laferrière.

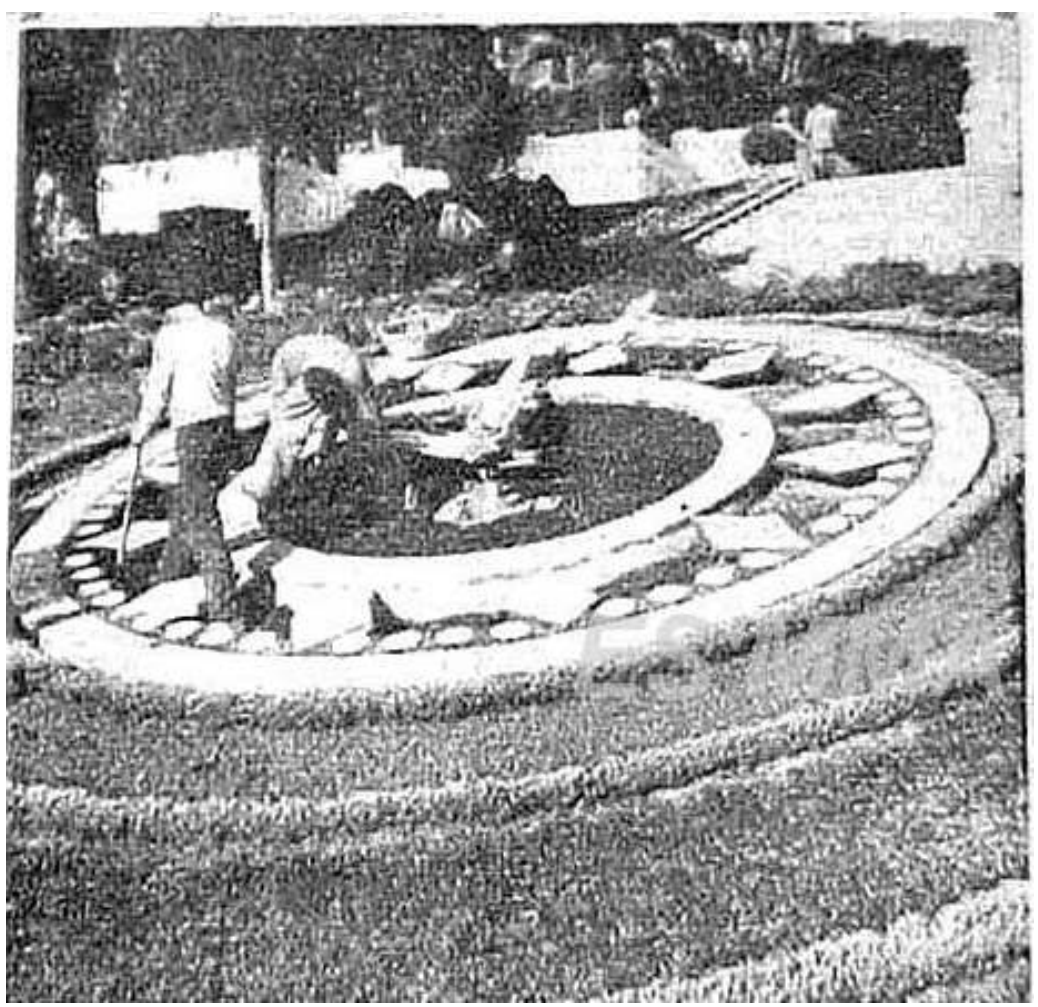

Nous pouvons avancer l'idée qu'avec le PAEE et le premier plan régional, Alger aurait pu voir l'émergence de la figure de l'architecte paysagiste, mais probablement de manière aussi ténue qu'en métropole à la même époque. Les architectes étaient alors réunis dans des sociétés professionnelles puissantes comme Les Amis d'Alger, la Société des architectes modernes et la Chambre syndicale des architectes diplômés par le gouvernement. Ils ont marqué de leur présence les grands événements de propagande de l'aménagement urbain colonial : les expositions de 1933 et 1936, et les projets du boulevard Laferrière. Pas plus qu'en métropole, la figure de l'architecte paysagiste n'a pu cependant être reconnue pendant l'entre-deux guerres (Le Dantec, 2002), la compétence paysagiste étant prise en charge par les métiers d'architecte, d'urbaniste et parfois d'ingénieur des Ponts et Chaussées. Cette reconnaissance internationale sera obtenue avec la création de la Fédération internationale des architectes paysagistes (IFLA) en 1947.

\section{Quasi disparition de l'idée de paysage sous les $2^{\mathrm{e}}$ et $3^{\mathrm{e}}$ plans régionaux}

Un deuxième plan régional d'Alger est élaboré entre 1938 et 1942 sous la responsabilité de l'ingénieur des Ponts et Chaussées Pierre Renaud, et sous la direction de l'architecte urbaniste Raymond Coquerel. Il livra plusieurs projets réalisés par des ingénieurs et architectes urbanistes tels que Rotival et Descoutures ${ }^{17}$. L'essentiel du plan concernait des travaux d'ingénierie de voiries, de gares maritime, ferroviaire et routière par l'architecte Urbain Cassan (1890-1979), d'aménagement du parc des sports des Tagarins et de la plage artificielle de Bab el-Oued des deux architectes Meunier et Tombarel. Zohra Hakimi (2011) indique que la ville d'Alger entre 1931 et 1942 a mobilisé tous ceux 
qui se réclament du titre d'architecte en affirmant que si la cohérence urbaine suppose, à une échelle spécifique, un agencement de compétences multiples, l'architecte peut prétendre, dans l'élaboration des plans d'urbanisme, synthétiser les savoirs d'autres professions.

Parallèlement au plan régional, Charles-Édouard Jeanneret-Gris, dit Le Corbusier (1887-1965), connu pour son " paysagisme bocager » (Le Dantec, 2002), élabore en 1942 un plan directeur pour le Grand Alger. Il illustre son plan par des croquis de jardins et d'espaces verts abritant ses unités d'habitation en forme de $Y$, mais cela n'a pas suffi à convaincre. On lui reprochait que les dimensions du construit fussent équivalentes ou supérieures à celles de l'espace vert (Maisonseul, 1942, cité par Hakimi, 2011). En cette période de guerre, ni le 2e plan régional ni le plan de Le Corbusier n'ont pu aboutir, laissant place à un troisième plan d'aménagement de la région d'Alger.

\section{L'urbanisme vert avec le troisième plan d'aménagement régional (1945-1953)}

37 La pression démographique et immobilière après la Seconde Guerre se traduit donc par un troisième plan d'aménagement régional, élaboré en 1948 par Jacques Wattez directeur du plan, l'urbaniste Henri Prost, l'architecte aquarelliste Jean de Maisonseul (1912-1999) et l'ingénieur des Ponts et Chaussées Eugène Colomb. Le plan indique des dispositifs de zones fonctionnelles (transports, industries, commerces, etc.) ainsi qu'une protection de la ceinture verte des coteaux. L'insertion de l'habitat dans la verdure sur les crêtes permet de bénéficier de l'air, du soleil et de la vue, des servitudes non altius tollendi, et d'un classement de sites naturels par la commission départementale des monuments naturels et des sites (dans Urbanisme, ${ }^{\circ} 7$, avril-maijuin 1952, cité par Hakimi, 2011), ce qui s'est manifesté par la création de jardins et d'espaces publics pour une population de 940000 projetée sur 30 ans (1976) (Deluz, 1988).

La dominance des architectes après 1940, l'émergence timide des paysagistes concepteurs en France et l'ampleur des programmes d'habitat jusqu'en 1953 avaient abouti à un urbanisme et à une création d'espaces extérieurs verts discutables, comme l'opération du Champ de Manœuvre (1951) de Bernard Zehrfuss et de ses collaborateurs architectes (Hakimi, 2011) ${ }^{18}$. Cette situation poussa les autorités à changer de méthode de travail et à créer l'Agence du plan qui réunit plusieurs compétences : sociologues, financiers, juristes, etc.

\section{La pratique paysagiste de Gérald Hanning à l'Agence du plan (1954-1962)}

L'urbaniste Pierre Dalloz dirige en 1954 l'Agence du plan ${ }^{19}$ nouvellement créée par la municipalité de Jacques Chevallier (1953-1959), et fait appel à l'architecte urbaniste Gérald Hanning (1919-1980) pour encadrer les travaux de l'Agence (Almi, 2002). Cet architecte dessine et organise les paysages urbains selon des projets inspirés de la culture traditionnelle (Culot et Thiveau, 1992) et des travaux de l'agronome Jean-Pierre Faure $^{20}$ (Almi, 2002)

Sa méthode, à l'échelle du territoire, expliquent Maurice Culot et Jean-Marie Thiveau, met en place une trame urbaine d'Alger sous forme de grille en unité géographique 
orientant les constructions et les espaces à aménager. Cette trame devient un outil d'interprétation et de composition du paysage urbain et rural. Gérald Hanning élabore un système d'occupation et de répartition $d u$ sol en îlots denses, en zones d'équipements et de jardins, qu'il formalise dans un plan avec des figures de haricots ou de doigts de gants, qui traduisent une occupation des crêtes, le franchissement d'une vallée ou la préservation d'un ravin.

On distingue ces pratiques dans l'opération des 26000 logements des Annassers, où Gérald Hanning qualifie l'espace urbain par un contraste entre «le côté rue » et «le côté jardin ». Il précise un plan d'épannelage qui reconstitue les courbes de niveau du site (Deluz, 1988). Mais sa méthode ne trouve pas d'interlocuteur dans les corps techniques représentés par les ingénieurs des Ponts et Chaussées. Ces derniers parlent de voiries, d'infrastructures et de lots à bâtir (Culot et Thiveau, 1992) et non de paysage et de qualité de cadre de vie des habitants.

\section{La fin de la perspective urbanistique paysagiste}

En raison en 1958 des effets du plan de Constantine ${ }^{21}$ (présence de nombreux bureaux d'études venus de France), de la nécessité d'une rapidité d'exécution et de la disparition des exigences d'organisation des espaces, une gigantesque machine administrative est mise en place en Algérie comme en métropole, privilégiant les objectifs quantitatifs sur les visées qualitatives (Le Dantec, 2002). Face à elle, l'Agence du plan, avec sa grille et ses préoccupations paysagistes, handicapée par une mise en œuvre lente fait pauvre figure. L'urbanisme français apparaît comme un « urbanisme d'architectes » (Culot et Thiveau, 1992). Jacques Chevallier démissionne en 1959 et Gérald Hanning se retire pour travailler dans la région parisienne. L'une des dernières initiatives des autorités françaises est le projet d'une nouvelle capitale à l'est d'Alger « Le Rocher Noir » (actuel Boumerdès) selon l'étude de l'architecte Home de Marien, mais vite transformé après l'indépendance de l'Algérie en 1962. S'ouvre alors une nouvelle ère pour l'Algérie postcoloniale, héritière d'un "urbanisme d'architectes" et d'un vaste chantier immobilier, commencé avec le plan de l'architecte urbaniste Charles Bachofen en 1964, qui gomme le travail paysagiste de Gérald Hanning (Deluz, 1988).

\section{Conclusion}

Malgré les multiples travaux de plantations et de replantations dus aux premières conquêtes des villes algériennes, l'espace urbain algérois semble ne pas avoir connu de figures de paysagistes concepteurs autonomes à l'époque coloniale, distinctes des ingénieurs et des architectes. Le travail de jardinage des premiers "planteurs» du génie qualifié de paysagisme militaire, créant des places, des squares, des jardins et des voies publiques selon des tracés régulateurs, et les tentatives artistiques des jardins et des parcs publics paysagers ont marqué les premières pratiques paysagistes urbaines inspirées du modèle métropolitain.

C'est après 1930 que les pratiques urbaines paysagistes commencent à faire une place à des professionnels, ingénieurs et architectes, de plus en plus autonomes, notamment avec René Danger, Tony Socard, Henri Prost et Gérald Hanning. Ces derniers adoptent les idées et les pratiques du paysagisme professionnel oscillant en France entre 
architecture de jardin et horticulture ornementale. Le travail de gestion de l'embellissement de l'espace public reste confié à des ouvriers jardiniers formés comme entrepreneurs et techniciens, ou autodidactes. L'Algérie, n'ayant pas subi de destruction pendant la Seconde Guerre mondiale, ne connaît pas la même période de reconstruction qu'en métropole, mais elle n'échappe pas, d'une part, à l'exode rural qui suppose d'intensifier la production de logements et, d'autre part, à la doctrine moderne qui considère les espaces urbains plantés comme de simples équipements fonctionnels ${ }^{22}$.

Le plan de Constantine, rédigé dans l'urgence, amorce en effet un urbanisme peu soucieux de l'embellissement réglementaire de type paysagiste. Toutefois, beaucoup de projets emblématiques (jardin d'Essai, boulevard Guillemin, etc.) ont vu le jour pendant cette période. Leurs tracés témoignent d'une connaissance certaine de l'ordonnancement et de la sensibilité de leurs concepteurs à l'art des jardins et à l'agencement des espaces publics. À l'aube de l'Indépendance, l'architecte paysagiste reste une figure émergente en Algérie et bien des espaces aménagés subsistent, charnières entre les périodes coloniales et les réalisations postcoloniales que nous analyserons dans un prochain article.

Remerciements à Pierre Donadieu pour la relecture de cet article.

\section{BIBLIOGRAPHIE}

Almi, S., Urbanisme et Colonisation : présence française en Algérie, Liège, Éditions Pierre Mardaga, 2002.

Béguin, F., Arabisances. Décor architectural et tracé urbain en Afrique du Nord, 1830-1950, Paris, Dunod, 1983.

Bennani, M., « Le Système des parcs et jardins publics du début du protectorat français au Maroc. Rabat le prototype de la ville idéale (1912-1930) ", thèse de doctorat en architecture et paysage, Paris, EHESS, 2006, $563 \mathrm{p}$.

Blanchon, B., «Les paysagistes français de 1945 à 1975 ", Les Annales de la recherche urbaine, n $^{\circ}$ 85, Paris, PUCA, 1999, p. 20-29.

Blanchon-Caillot, B., « Pratiques et compétences paysagistes dans les grands ensembles d'habitation, 1945-1975 », Strates, $n^{\circ} 13$, 2007, mis en ligne le 5 novembre 2008, URL : http:// strates.revues.org/5723.

Carra, P., Gueit, M., « Le jardin d'essai du Hamma », Gouvernement général de l'Algérie, direction de l'Agriculture, Alger, 1952.

Chabbi-Chemrouk, N., " Jardins et parcs publics d'Alger aujourd'hui », dans Cohen, J.-L., Oulebsir, N., Kanoun, Y. (dir), Alger paysage urbain et architectures, 1800-2000, Paris/Besançon, Les Éditions de l'imprimeur, 2003.

Chaux, R., « La formation du paysagiste », Voir en Yvelines, $\mathrm{n}^{\circ}$ 3, Versailles, CAUE des Yvelines, 1989, p. 40-41. 
Cice, C. et Dubost, F., « La profession de paysagiste ", rapport d'une enquête financée par la mission de la recherche urbaine, Centre de sociologie des arts, non publié, 1986.

Cohen, J.-L., « Henri Prost, des grands paysages aux métropoles » dans « Henri Prost et le plan directeur d'Istanbul 1936-1951 », exposition virtuelle mise en ligne en janvier 2012.

Culot, M., et Thiveau, J.-M., Architectures françaises Outre-Mer, Liège, Mardaga, 1992.

Deluz, J.-J., L'Urbanisme et l'Architecture d'Alger : aperçu critique, Liège, Éditions Pierre Mardaga, 1988.

Donadieu, P., « Les paysagistes dans le monde : les paysagistes en Algérie », Topia, URL : http:// www.topia.fr/_chroniques, 2014.

Donadieu, P., Les Paysagistes ou les Métamorphoses du jardinier, Arles/Versailles, Actes Sud/ENSP, Versailles, 2009.

Donadieu, P., « Le paysage, les paysagistes et le développement durable : quelles perspectives ? », Économie rurale, $\mathrm{n}^{\circ}$ 297-298, janvier-avril 2007, mis en ligne le 1er mars 2009, URL : http:// economierurale.revues.org/1923.

Donadieu, P., « Entre urbanité et ruralité », Les Annales de la recherche urbaine, n 85, Paris, PUCA, 1999, p. 6-15.

Dubost, F., «Les paysagistes et l'invention du paysage », Sociologie du travail, vol. $35, \mathrm{n}^{\circ} 4$, octobredécembre 1983, p. 432-445.

Fédération française du paysage, «Paysagiste concepteur : définition de la profession et de ses modalités d'exercice », Afnor, octobre 2009.

Hakimi, Z., Alger politiques urbaines 1846-1958, Saint-Denis, Éditions Bouchène, 2011.

Laing Meason, G., On the landscape architecture of the great painters of Italy, London, C. Hullmandel's Lithographic Establishment, 1828.

Laprade, A., "L'urbanisme en Afrique du Nord, 20 ans d'urbanisme appliqué », L'Architecture d'aujourd'hui, n 3, mars 1939.

Le Dantec, J.-P., Le Sauvage et le Régulier : art des jardins et paysagisme en France au $\mathrm{XX}^{e}$ siècle, Paris, Le Moniteur, 2002.

Maisonseul, J. de, El Djazaïr, revue du ministère du Tourisme algérien, nº 6, 1942.

Malverti, X., et Picard, A., Les Villes coloniales fondées entre 1830 et 1870 en Algérie : les tracés de ville et le savoir des ingénieurs du génie, Grenoble, École d'architecture de Grenoble, 1989.

Oulebsir, N., Les Usages du patrimoine : monuments, musées et politique coloniale en Algérie (1830-1930), Paris, Éditions de la Maison des sciences de l'homme, 2004.

Racine, M., (dir.), Créateurs de jardins et de paysages en France du XIX ${ }^{e}$ siècle au XXI ${ }^{e}$ siècle, Arles/ Versailles, Actes Sud/ENSP, 2002.

Royer, J. et al., L'urbanisme aux colonies et dans les pays tropicaux, communications et rapports du Congrès international de l'urbanisme aux colonies et dans les pays de latitude intertropicale, t. II, Paris, Éditions d'Urbanisme, 1935.

Périodiques

Journal général des travaux publics et du bâtiment. Le Plan régional du grand Alger et l'aménagement du boulevard Laferrière, Maréchal Foch et des Tagarins : l'aménagement du boulevard Maréchal-Foch, $\mathrm{n}^{\circ}$ 1033, 24 septembre 1935. 
Journal général des travaux publics et du bâtiment. Le Plan régional du grand Alger et l'aménagement du boulevard Laferrière, Maréchal Foch et des Tagarins : Pour mettre en valeur les hauts de la ville, $\mathrm{n}^{\circ} 1034$, 27 septembre 1935.

Journal général des travaux publics et du bâtiment. Les grands travaux d'urbanisme à Alger, $\mathrm{n}^{\circ}$ 959, 8 janvier 1935.

Journal général des travaux publics et du bâtiment. La création et la transformation de jardins publics à Alger, $\mathrm{n}^{\circ}$ 864, 9 février 1934.

L'Afrique du nord illustrée. Le Jardin d'Essai du Hamma, nº 278, 11 avril 1914.

\section{NOTES}

1. Dans ce texte, le mot paysagiste (landscaper), employé seul, regroupe tous les métiers et formations du paysagisme (jardinier, entrepreneur, architecte paysagiste, concepteur paysagiste, ingénieur paysagiste, paysagiste planificateur, etc.).

2. Il s'agit de l'ENSP de Versailles, l'ENSAP de Bordeaux, l'ENSAP de Lille, l'ENP de Blois (exENSNP), Agrocampus ouest d'Angers (ex-INH), l'ESAJ de Paris et l'ITIAPE de Lille.

3. Le landscape gardener ou jardinier paysagiste désigne le technicien de jardin public et privé chargé de l'entretien et parfois de la conception et de la réalisation (Donadieu, 2009).

4. Des professionnels organisés entre autres dans la Fédération française du paysage (FFP), l'Union nationale des entrepreneurs paysagistes, l'Association des paysagistes-conseils de l'État et l'Association des ingénieurs paysagistes des villes.

5. Arrêté du 27 août 1946 portant organisation de la section du paysage et de l'art des jardins à l'École nationale d'horticulture de Versailles, Journal officiel de la République française du 1er septembre 1946, p. 7605.

6. Décret $n^{\circ}$ 2014-1400 du 24 novembre 2014 portant création du diplôme d'État de paysagiste et fixant les conditions de recrutement par concours et de formation des étudiants.

7. Loi du 14 mars 1919 complétée en 1924, imposant les plans d'aménagement, d'embellissement et d'extension (PAEE) aux villes françaises de plus de 10000 habitants, ou à celles présentant un caractère pittoresque, artistique ou historique signifiant. Journal officiel de la République française, art. 1, du 15 mars 1919, p. 2726.

8. À Sidi Bel Abbès, Ténès, Orléansville, Mascara et Sétif, les essences d'arbres changeaient selon les différents espaces urbains (places, voies, squares, etc.), avec le soin porté à l'emplacement des plantations, kiosques, promenades, monuments, fontaines, etc. Les ingénieurs du génie déménagent dans certains cas des édifices militaires pour créer une promenade le long du front de mer ; élargissent des voies étroites; déplacent des portes de ville de quelques mètres afin de faire profiter une place de la vue sur la campagne. Dans certains cas les propriétaires sont contraints de planter et d'assurer l'entretien des arbres sur le trottoir (Malverti, 1989).

9. Décret-loi du 25 juillet 1935 qui institue les projets régionaux d'urbanisme, Journal officiel de la République française du 27 juillet 1935, p. 8154.

10. Claro fait la jonction entre différents monuments, les mosquées Hanafites, Malikites et le palais consulaire, par un ensemble de jardins agrémentés de cours et de portiques (Hakimi, 2011). 11. En application des lois des 14 mars 1919 et 19 juillet 1924 qui stipulent la participation aux jurys des concours de conceptions architecturales et urbaines d'autres services de l'État tels que les Beaux-Arts, les monuments historiques, plus tard ceux de l'agriculture et les transports.

12. Les parcs paysagers sont des jardins aux tracés irréguliers avec bosquets, comportant au XIX siècle des fausses grottes avec cascades, des rivières sinueuses, des fabriques (ponts, belvédères, temples, etc.) et en général de nombreuses essences exotiques disposées sur de vastes pelouses. 
13. Une réglementation assez souple qui utilise le terme large d'artiste paysagiste, sous condition de joindre une liste de références et une copie des diplômes et titres des concurrents, Journal général des travaux publics et du bâtiment, $\mathrm{n}^{\circ} 864,1934$.

14. Maurice Rotival, en tant que conseiller municipal, programme, dessine les aménagements et participe de fait au concours avec Jacques Guiauchain. Cependant la presse de l'époque attribue le projet à Rotival, Journal général des travaux publics et du bâtiment, n 959, 1935.

15. Journal général des travaux publics et du bâtiment, $\mathrm{n}^{\circ} \mathrm{s} 1033$ et $1034,1935$.

16. Il s'agit des écoles d'agriculture de Philippeville (actuel Skikda) (1900), de Sidi-Bel-Abbès (1930), d'Aîn-Témouchent (1929), de Guelma (1922), des Mechtras (Boghni) (1930) ainsi que des deux Écoles d'horticulture et ménagère agricole du jardin d'Essai (Alger) (1918).

17. On peut apercevoir certains exemples de cette période à travers les dessins de J. Descoutures pour son plan d'aménagement de la ville nouvelle d'El Alia (Voir Laprade, 1939).

18. La croissance de la commande publique pendant cette période augmente et l'absence de paysagistes a fait que les architectes se sont peu souciés du traitement des espaces extérieurs (Dubost, 1983). Voir aussi à ce sujet les travaux de Bernadette Blanchon-Caillot, 2007.

19. L'agence composée d'urbanistes, d'architectes, d'ingénieurs, de sociologues, de géographes, de financiers et de juristes agissait comme conseiller des services officiels de l'urbanisme de la commune et du département, et comme conseiller direct du maire (Deluz, 1988).

20. Faure côtoie les architectes adeptes de la charte d'Athènes. Ses travaux sur la densité urbaine inspireront Hanning (Almi, 2002).

21. Du point de vue urbain, le plan se résume en un vaste programme d'aménagement et de construction de logements (50.000 par an dont 10.000 à Alger) sur le territoire algérien, (Culot et Thiveau, 1992).

22. Voir les travaux de Bernadette Blanchon (1999, p. 20-29), au sujet des pratiques paysagistes dans les grands ensembles entre 1945 et 1975.

\section{RÉSUMÉS}

La profession d'architecte paysagiste est inexistante aujourd'hui en Algérie. En dépit des multiples projets emblématiques d'aménagement paysager qui ont vu le jour, depuis les premières plantations du génie militaire français jusqu'au plan de Constantine, la figure du concepteur paysagiste peine à voir le jour en Algérie, contrairement aux deux pays voisins (le Maroc et la Tunisie). En partant d'une démarche historique, cet article montre comment les compétences de l'ingénieur ou de l'architecte ont été sollicitées pour réaliser des projets d'aménagement paysager à Alger jusqu'en 1962.

The profession of landscape architect does not exist in Algeria today. In spite of the many emblematic landscape development projects that have seen the light of day in Algeria, ranging from the first plantations by French military engineers to the master plan of the city of Constantine, the figure of the landscape designer had difficulty in emerging in Algeria compared with the neighbouring countries of Morocco and Tunisia. Based on an historical approach, this article shows how the figure of the engineer or architect conducting landscape developing projects in Algiers nevertheless emerged up until 1962. 
INDEX

Keywords : landscape architects, urban development, Algiers, French colonisation

Mots-clés : architectes paysagistes, aménagement urbain, Alger, colonisation française

\section{AUTEURS}

\section{FARES TRODI}

Fares Trodi est enseignant-chercheur en urbanisme et doctorant à l'École polytechnique d'architecture et d'urbanisme (EPAU, Alger, Algérie).

ftrodi2000[at]yahoo[dot]fr

\section{NADIA DJELAL}

Nadia Djelal est professeure en géographie et urbanisme à l' EPAU. nadiadjelal[at]gmail[dot]com

\section{PHILIPPE POTIÉ}

Philippe Potié est professeur à l'École nationale supérieure d'architecture de Versailles (ENSAVersailles, France), Laboratoire de recherche de l'école d'architecture de Versailles (LéaV, Versailles, France).

philippe.potie[at]versailles.archi[dot]fr 